\title{
Special issue dedicated to Heinz Hoffmann
}

\author{
Heinz Rehage $^{1} \cdot$ Michael Gradzielski $^{2}$
}

Published online: 14 September 2015

(C) Springer-Verlag Berlin Heidelberg 2015

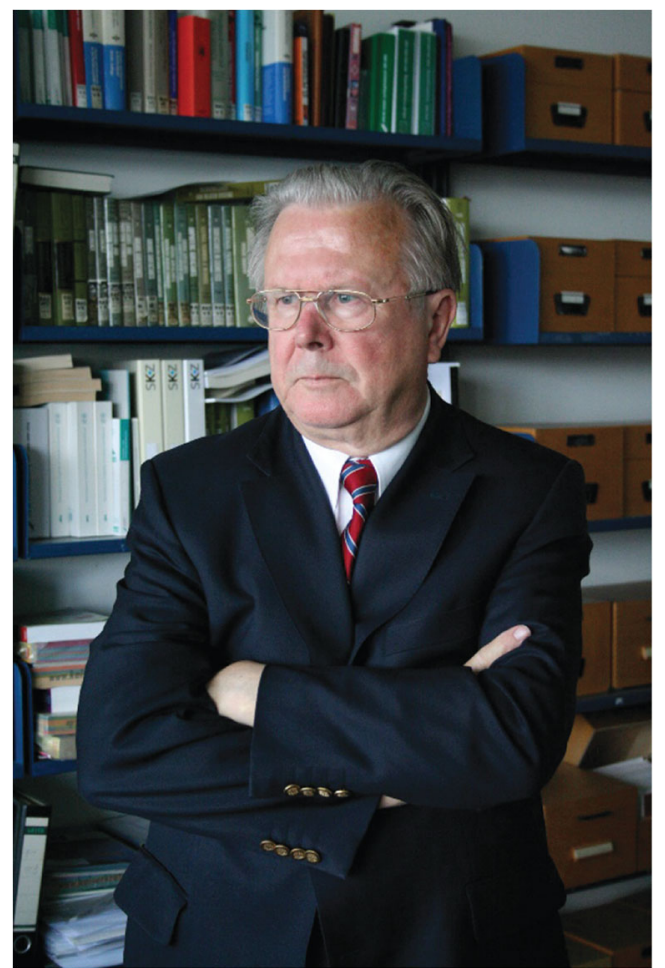

Heinz Rehage

heinz.rehage@tu-dortmund.de; http://www.pcii.chemie.unidortmund.de

Michael Gradzielski

michael.gradzielski@tu-berlin.de

1 Fakultät für Chemie und Chemische Biologie, Lehrstuhl für Physikalische Chemie II, Technische Universität Dortmund, Otto-Hahn-Straße 6, 44227 Dortmund, Germany

2 Stranski-Laboratorium für Physikalische Chemie und Theoretische Chemie, Institut für Chemie, Technische Universität Berlin, Strasse des 17. Juni 124, 10623 Berlin, Germany
This special issue is dedicated to Heinz Hoffmann on the occasion of his 80th birthday (23 June 2015). This special date also marks the 40th anniversary of the chair of Physical Chemistry I at the University of Bayreuth where Heinz Hoffmann and his group started his research in the area of colloid and interface science. At 4th of July, numerous former coworkers, colleagues and friends from all over the world celebrated these special events in Bayreuth with a very nice scientific symposium. As one of the outstanding persons in the field of colloid and interface science, Heinz Hoffmann has a large family of friends from all over the world and many of them contributed to this special issue.

Heinz Hoffmann was born in June 1935 in Käshofen/Pfalz (Germany). After finishing his schooling in 1955, he began to study chemistry at the University in Würzburg. Two years later, he moved to the 'Technische Hochschule Karlsruhe' where he received his master degree in physical chemistry. Subsequently, he started his research career and in 1962 he finished his $\mathrm{PhD}$ in electrochemistry under the supervision of Prof. Walther Jaenicke. Afterwards, Heinz Hoffmann went for a post-doc at the 'Case Western Reserve University' in Cleveland/Ohio where he spent 1 year with Professor E. Yeager. In this time, he developed many new ideas concerning the measurements of fast chemical reaction kinetics. Special emphasis was drawn on the development of new relaxation techniques. It was during this postdoctoral visit in Cleveland that Heinz Hoffmann met and married Claudia, his charming wife, who remained an anchoring point in his busy life ever after.

After returning to Germany, Heinz Hoffmann worked for 1 year as a research and teaching assistant at the University of Erlangen. Afterwards, he returned to the Case Western Reserve University in Cleveland where he developed new fields of activity. In 1969, he finally returned to the University of Erlangen where he completed his habilitation on the topic 
'kinetic investigations of octahedral nickel complex formations'. During this period, he started to build up a new laboratory for kinetic investigations, and he used advanced techniques such as shock-wave tubes or pressure- and temperature-jump experiments. Having become an expert in this set of experiments, a few years later, he used these methods to study the aggregation processes of surfactant micelles - thereby starting his scientific career in the field of colloid science. Close inspection of these phenomena revealed that two different kinetic processes occurred in surfactant solutions. The fast relaxation time could be explained by the exchange rates of the surfactant molecules, which were permanently leaving or entering the micelles. The long relaxation time was attributed to the limited average lifetime of the whole micellar aggregates. Due to these detailed investigations, Heinz Hoffmann became one of the pioneers in the field of micellar kinetics. In international collaborations and friendships with Raoul Zana, Gunnar Anianson and Stefan N. Wall, a theoretical model was derived which allowed the quantitative description of the dynamic aggregation processes. From this theory, it was finally possible to understand micellar kinetics and relate this understanding to the calculation of many interesting micellar properties, such as the mean value of the aggregation number, the width of the micellar distribution or the average aggregation numbers of the micellar nuclei.

In recognition of his scientific success, Heinz Hoffmann was appointed to a full professor position for Physical Chemistry in Bayreuth (1975), and 1 year later, he was honoured with the prestigious 'Nernst-Haber-Bodenstein' award of the 'Deutsche Bunsengesellschaft'. After such a successful starting time, Heinz Hoffmann broadened his scope, covered new fields of activity and his group developed into one of the major institutes for fundamental research in surfactant science. During the next years, he worked on many different subjects, such as viscoelastic surfactant solutions, vesicles, liquid crystals and ringing gels. As an excellent scientist, he always tried to find a reasonable way of interpreting the experimental data; introducing some simplifications, when necessary, but keeping the essential features. An extended analysis of his broad spectrum of scientific contributions shows the major source of Heinz Hoffmann's success: he can easily combine different results to get an unexpected solution. It is very characteristic for him to use different types of experimental techniques, in particular electron microscopy, small-angle neutron scattering, rheology, electric birefringence, static and dynamic light scattering, flow birefringence and optical microscopy for experimental investigations of complicated colloidal systems. From this large amount of information, he has the talent to separate the most important phenomena. In this way, he has pioneered many areas of surfactant science, and very often he succeeded in delivering key results on which present knowledge has been built. During the last
20 years, he investigated a large number of different colloidal systems, such as microemulsions, ringing gels, networks, liquid crystals, viscoelastic foams, multilamellar vesicles iridescent phases and clay colloids and he developed new formulation techniques. On all these topics, he has helped to clarify the relations between the molecular structure of amphiphiles, their mesoscopic organization and the properties and dynamic features of these systems resulting from them. Of particular and long-standing interest to him was the investigation of the $\mathrm{L}_{3}$-sponge phase, a type of self-assembled structure that has puzzled scientist for some while. In recent years, Heinz Hoffmann has developed special techniques to build up defined suspensions of multilamellar, onion-like vesicles. In that context, the aggregates were finally formed by chemical reactions and not by the application of external shear forces, which are more complicated to control. In all these areas, his work is still making a significant impact on surfactant science. His fundamental research contributions are published in more than 380 papers, many book articles and patents. His scientific experience, his advice and views continue to be highly esteemed. The interesting results led to many international collaborations and friendships with colleagues in many different countries. In 1984, Heinz Hoffmann was a visiting scientist at the Du Pont Company in Wilmington (Delaware), and in 1989, he was invited to spend several months at the 'Tokyo Science University' in Japan. Over decades, Heinz Hoffmann was a dedicated member of the editorial board of several scientific journals, dealing with colloid science. He has organized many national and international conferences and it is certainly a hallmark of his career that he served as a founding chairman of the European Colloid and Interface Science (ECIS), and for many years, he was elected as the general secretary of this society (1987-2000). In addition to these positions, Heinz Hoffmann was also the president of the German Colloid Society from 1987 to 1991. On behalf of his outstanding contributions to colloid chemistry, Heinz Hoffmann received the 'Wolfgang-OstwaldPrize' of the German Colloid Society (1995), the 'Lectureship Award' from the Chemical Society of Japan and India (1998), and finally the Overbeek gold medal of the ECIS in 2011. He was also instrumental in establishing the Bayreuth Center for Colloids and Interfaces (Bayreuther Zentrum für Kolloide und Grenzflächen, BZKG) in 2000, which guaranteed that Bayreuth remains a scientific centre in the field of colloid and interface research. In spite of all these different activities, his friends, colleagues and students are deeply impressed by his kindness and modesty and they know him as a warm and generous person.

Even after his official university retirement in 2003 Heinz Hoffmann remained very active in science. He started an independent science lab and moved towards the more applied aspects of colloid science, but never forgetting to connect even 
relatively complex systems to fundamental physical chemistry. In this time, he continued and intensified cooperations with Japanese and Mexican companies working in colloid science, amongst other industry collaborations that he was pursuing. This time outside of the university was very productive and yielded many interesting contributions to the fields of solubilization (for instance HIPME: high internal phase microemulsion), hydrogels or the stabilization of protein emulsions. A very interesting finding was certainly the extreme swelling of lamellar phases that is observed upon admixing solvents like glycerol to aqueous solutions, which Heinz Hoffmann could explain by the very fundamental phe- nomenon of vanishing 'van-der-Waals attraction', as the refractive indices of dispersed lamellae and solvents became very close.

We sincerely do hope that Heinz Hoffmann will enjoy health and happiness for many years to come. The present issue may serve as inspiration source and describes the continuation of many interesting projects, which were first proposed and investigated by Heinz Hoffmann and his coworkers.

Michael Gradzielski Heinz Rehage

TU Berlin TU Dortmund 